\title{
Neurofibromatosis Type 1 with Highly Active Relapsing-Remitting Multiple Sclerosis (RRMS)
}

\author{
Silvia Ciotti ${ }^{1}$, Antonella Cometa ${ }^{2}$, Claudia De Carlo ${ }^{3}$, Giancarlo Martini ${ }^{4}$, Andrea Marona ${ }^{5}$, Laura Filippetti ${ }^{1}$, \\ Diego Carducci ${ }^{6}$, Silvano Baratta ${ }^{1}$, Mauro Zampolini ${ }^{6}$, Francesco Corea ${ }^{6}$ \\ ${ }^{1}$ USL Umbria 2, Department of Rehabilitation, S.C.R.I.N. Trevi, Italy \\ ${ }^{2}$ USL Umbria 2, Department of Rehabilitation, S.M. Stella, Orvieto, Italy \\ ${ }^{3}$ USL Umbria 2, Department of Rehabilitation, Domus Gratiae, Terni, Italy \\ ${ }^{4}$ USL Umbria 2, Department of Rehabilitation, Ospedale di Cascia, Italy \\ ${ }^{5}$ USL Umbria 2, Department of Neuroradiology, Ospedale S.G. Battista di Foligno, Italy \\ ¿USL Umbria 2, Department of Rehabilitation and Neurology, Ospedale S.G. Battista di Foligno, Italy
}

\section{Doi: 10.12890/2021_002190 - European Journal of Case Reports in Internal Medicine - ( $E$ EFIM 2021}

Received: 06/12/2020

Accepted: $23 / 12 / 2020$

Published: 03/02/2021

How to cite this article: Ciotti S, Cometa A, De Carlo C, Martini G, Marona A, Filippetti L, Carducci D, Baratta S, Zampolini M, Corea F. Neurofibromatosis type 1 with highly active relapsing-remitting multiple sclerosis (RRMS). EJCRIM 2021;8: doi:10.12890/2021_002190.

Conflicts of Interests: FC received travel grants and modest honoraria for membership of advisory boards from Almirall, Sanofi, Merck and Biogen Acknowledgements: Editorial assistance was provided by Francesca Cappellini, PhD, and Aashni Shah (Polistudium SRL, Milan, Italy).

This article is licensed under a Commons Attribution Non-Commercial 4.0 License

\section{ABSTRACT}

Neurofibromatosis type 1 (NF1) is an autosomal dominant neurocutaneous disease which confers an increased risk of malignant tumour development. Relapsing remitting multiple sclerosis (RRMS) is an inflammatory demyelinating disease of the central nervous system. The coexistence of multiple sclerosis and NF1 is rare but has been reported. Here, we describe the case of a 31-year-old man with NF1 and subacute walking problems with proximal pain in the lower limbs who was successfully treated with natalizumab.

\section{LEARNING POINTS}

- The coexistence of multiple sclerosis (MS) and neurofibromatosis type 1 (NF1) is very rare but has been described in the literature.

- Follow-up of patients with NF1 is important as the early detection and management of MS can prevent further disability.

- Appropriate treatment and physical therapy can improve the patient's activity and social life.

\section{KEYWORDS}

Multiple sclerosis, neurofibromatosis 1, neurorehabilitation, natalizumab, case report

\section{INTRODUCTION}

Neurofibromatosis type 1 (NF1) is an autosomal dominant neurocutaneous disease caused by NF1 gene mutations. Clinical manifestations include café-au-lait spots, Lisch nodules on the surface of the iris, freckles in the armpits or groin, and multiple neurofibromas on the peripheral and central nerves. Multiple sclerosis (MS) is a common inflammatory demyelinating disease of the central nervous system. The coexistence of MS and NF1 is rare but has been reported in the literature ${ }^{[1-3]}$. Here, we describe the a case of 31-year-old man with a diagnosis of highly active relapsing remitting multiple sclerosis (RRMS) and NF1. 


\section{CASE DESCRIPTION}

We describe the case of a 31-year-old man with a diagnosis of MS and NF1. His mother had NF1 but there was no family history of autoimmune disease. The patient had presented the typical cutaneous stigmata since childhood (Fig. 1).

In April 2019, the patient experienced subacute walking problems with proximal pain in the lower limbs. Spastic paraparesis quickly worsened and the patient was admitted to hospital. Urgent magnetic resonance imaging (MRI) of the brain with contrast enhancement showed multiple supra- and infra-tentorial demyelinating lesions (Fig. 2). In addition, multiple focal alterations with both centromedullary and peripheral distribution in C1, C2, C5 and D3 in the cervical and dorsal spinal cord were seen.
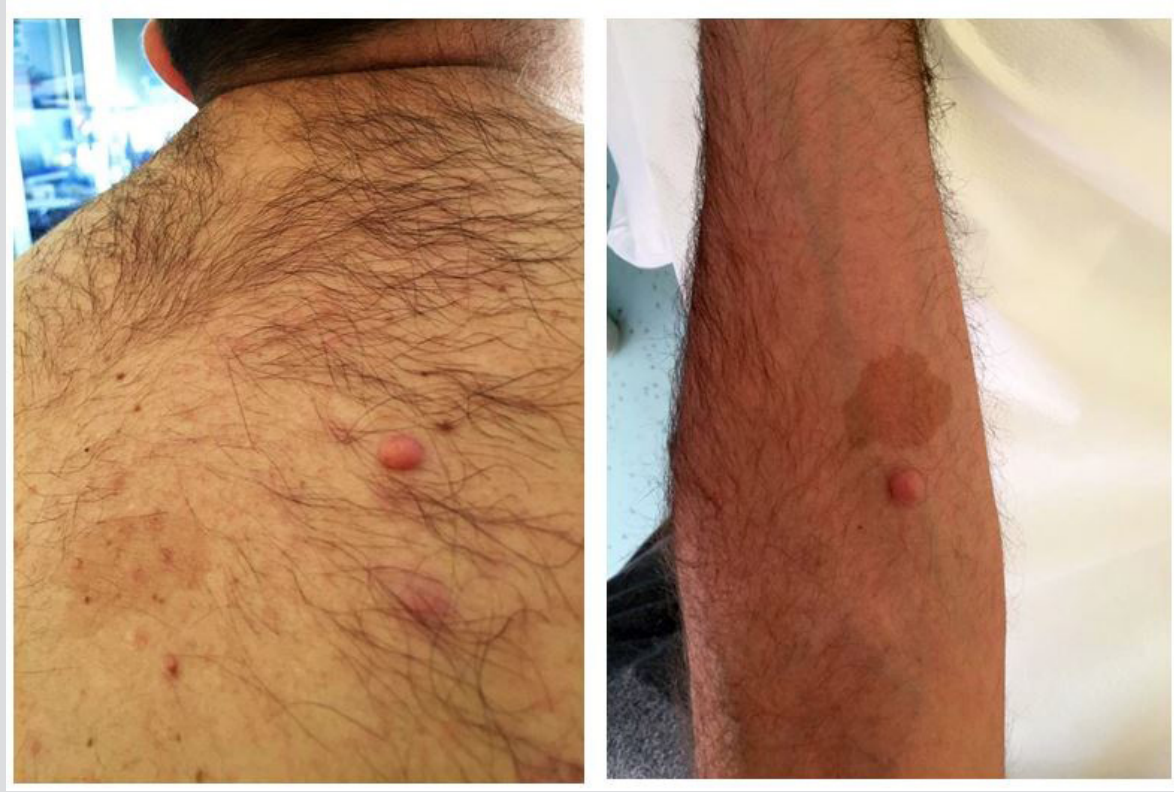

Figure 1. Café-au-lait spots, freckles and multiple neurofibromas on the arm and trunk, typical of neurofibromatosis type $1 a$

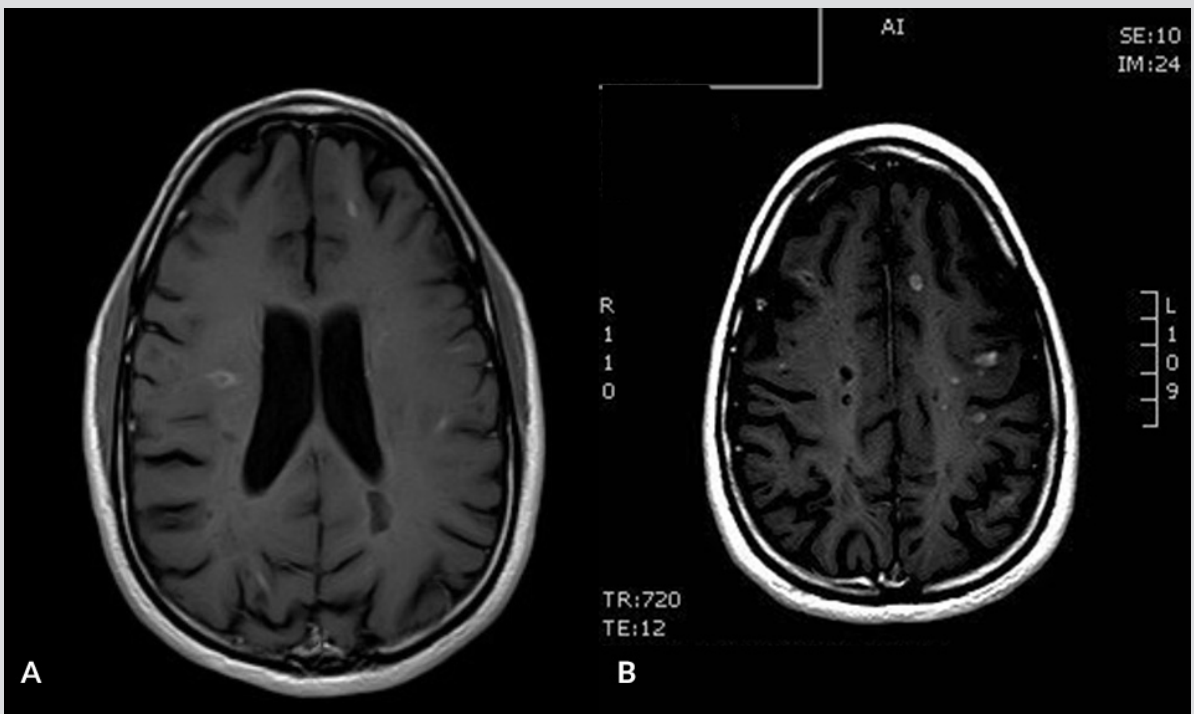

Figure 2. (A, B) Contrast enhanced MRI with T1-weighted sequences showing multiple active bi-hemispheric supratentorial lesions

A course of intravenous steroid therapy with methylprednisolone $1000 \mathrm{mg} /$ day for 5 days improved walking, which, however, remained slightly ataxo-spastic. A lumbar puncture demonstrated the presence of multiple oligoclonal IgG bands in the cerebrospinal fluid with a Link's index of 0.6. A formal diagnosis of MS was made according to the revised McDonald criteria of $2017^{[4]}$. Therapy with intravenous natalizumab 300 mg every 6 weeks was started within 2 months of symptom onset. The delayed infusion protocol was chosen because of an elevated JC virus index of 3.40 which indicated a potential risk of progressive multifocal leukoencephalopathy (PML). 
The patient had an Expanded Disability Status Scale (EDSS) score of 4.0. After 1 year of therapy, he had improved greatly and had an EDSS of 2.5 as well as complete absence of disease activity on imaging.

\section{DISCUSSION}

The coexistence of MS and NF1 is uncommon but challenging for the physician. To our knowledge no other patient with NF1 and RRMS has been treated with natalizumab. An association between NF1 and an increased incidence of autoimmune disease, such as MS, is possible, as described in a recent French study ${ }^{[1]}$.

NF1 is a clinically heterogeneous genetic neurocutaneous disease characterized by café-au-lait spots (Fig. 1), Lisch nodules on the surface of the iris, armpit or groin freckles, and multiple neurofibromas at various sites with risk of evolution into malignant tumours. NF1 is caused by mutations in the tumour suppressor gene NF1 (neurofibromin 1; 17q11.2) and rarely by 17q11 microdeletion (5\% of cases).

The overall risk of NF1 patients developing malignancy is $10-12 \%$ and higher than that of the general population. In northeast Italy, the chance of having both NF1 and MS is greater than would be expected based on the prevalence rates of the two diseases ${ }^{[2]}$. Some of our NF1 patients have ancestors with both these conditions.

Another study that investigated the relationship between MS and NF1, indicated a higher risk of MS among patients with NF1, and the possible development of different forms of MS (primary progressive, secondary progressive and relapsing-remitting) ${ }^{[3]}$. In a case series, three patients with primary progressive MS and NF1 had genetic polymorphisms and NF1 mutations associated with spastic paraplegia ${ }^{[5]}$. Lesions of the central nervous system due to MS and lesions of the peripheral nervous system due to neurofibromas both result in paraparesis and an increased risk of malignancy. Furthermore, skin lesions caused by NF1 also have a psychological impact due to their appearance, which can severely limit the patient's participation in social life. Cutaneous lesions may also discourage drug administration by injection.

This case report is important because it highlights the difficulty of choosing immunomodulatory therapy to ensure a reduction in central nervous system lesions while avoiding an oncogenic effect on NF1. Natalizumab therapy resulted in excellent disease control in this patient with a 2 point reduction in the disability scale, while minimizing the risk of possible oncogenic effects on NF1 triggered by immunomodulants and/or immunosuppressants. Due to the elevated JC virus index and the potential risks of PML, other disease-modifying treatments may be need to be altered in the short-term. This will expose patients to a severe risk of MS rebound and challenge the physician decision-making process $^{[6]}$.

Frequent and careful follow-up of patients with NF1 is needed, as early detection and management of MS is required to prevent further disability. It is also important to halt the progression of disability and improve activity and participation in social life with an individual rehabilitation program that takes account of neuromotor and psychological aspects.

\section{REFERENCES}

1. Bergqvist C, Hemery F, Ferkal S, Wolkenstein P. Neurofibromatosis I and multiple sclerosis. Orphanet J Rare Dis 2020;15(1):186.

2. Perini P, Gallo P. The range of multiple sclerosis associated with neurofibromatosis type 1. J Neurol Neurosurg Psychiatry 2001;71(5):679-681.

3. Iwanowski P, Kowalska M, Prendecki M, Dorszewska J, Kozubski W, Rydzanicz M, et al. Primary progressive multiple sclerosis and neurofibromatosis type 1. Mult Scler Relat Disord 2019;32:66-69.

4. Thompson AJ, Banwell BL, Barkhof F, Carroll WM, Coetzee T, Comi G, et al. Diagnosis of multiple sclerosis: 2017 revisions of the McDonald criteria. Lancet Neurol 2018;17(2):162-173.

5. Etemadifar M, Fatehi F, Sahraian MA, Borhanihaghighi A, Ardestani PM, Kaji-Esfahani M, et al. Multiple sclerosis and neurofibromatosis type 1: report of seven patients from Iran. Mult Scler 2009;15(9):1126-1130.

6. laffaldano P, Lucisano G, Pozzilli C, Brescia Morra V, Ghezzi A, Millefiorini E, et al. Italian iMed-Web database. Fingolimod versus interferon beta/glatiramer acetate after natalizumab suspension in multiple sclerosis. Brain 2015;138(Pt 11):3275-3286. 